\title{
A remark on divisibility of definable groups
}

\author{
Mário J. Edmundo * \\ CMAF Universidade de Lisboa \\ Av. Prof. Gama Pinto 2 \\ 1649-003 Lisboa, Portugal \\ edmundo@cii.fc.ul.pt
}

September 4, 2005

\begin{abstract}
We show that if $G$ is a definably compact, definably connected definable group defined in an arbitrary o-minimal structure, then $G$ is divisible. Furthermore, if $G$ is defined in an o-minimal expansion of a field, $k \in \mathbb{N}$ and $p_{k}: G \longrightarrow G$ is the definable map given by $p_{k}(x)=x^{k}$ for all $x \in G$, then for all $x \in G$, we have $\left|\left(p_{k}\right)^{-1}(x)\right| \geq k^{r}$ where $r>0$ is the maximal dimension of abelian definable subgroups of $G$.
\end{abstract}

${ }^{*}$ With partial support from the FCT (Fundação para a Ciência e Tecnologia), program POCTI (Portugal/FEDER-EU). MSC: 03C64; 20E99. Keywords and phrases: O-minimal structures and definable groups. 


\section{On divisibility of definable groups}

We work over an arbitrary o-minimal structure $\mathcal{N}$ and definable means $\mathcal{N}$ definable (possibly with parameters). We are interested here in understanding the subset $\left(p_{m}\right)^{-1}(x)$ of a definably compact definably connected definable group $G$ where $x \in G$ and, for each $m \in \mathbb{N}, p_{m}: G \longrightarrow G$ is the definable map that sends $z$ to $z^{m}$.

A definable group is a group whose underlying set is a definable set and the graphs of the group operations are definable sets. The notion of definably compact is the analogue of the notion of semi-algebraically complete and was introduced Peterzil and Steinhorn in [8]. The theory of definable groups, which includes real algebraic groups and semi-algebraic groups, began with Anand Pillay's paper [12] and has since then grown into a well developed branch of mathematics (see for example [8], [9] and [10]). In the literature there are many interesting results about definable groups which have an analogue in the theory of Lie groups. Among these we have the following properties:

(TOP) every definable group $G$ has a unique definable topological structure such that the group operations are continuous and the definable homomorphisms are also continuous;

(DCC) the descending chain condition for definable subgroups of a definable group $G$;

(QT) existence in the category of definable groups of the quotient of a definable group by a definable normal subgroup together with the existence of a corresponding definable section;

(AB) every definable group $G$ of positive dimension has a definable abelian subgroup of positive dimension.

Properties (TOP), (DCC) and (AB) were proved in [12]. Property (QT) is from [2]. In the paper [13] by Strebonski the following property is proved using the o-minimal Euler characteristic for definable sets defined by van den Dries [1] using the cell decomposition theorem. This is an Euler-Grothendieck characteristic for the definable category in the sense of [6].

(TOR) If $G$ is a definable group, then for all $m \in \mathbb{N}$, the subgroup $G[m]$ of 
$m$-torsion points of $G$ is a finite definable subgroup.

Property (TOR) has the following consequence:

(DIV) If $G$ is a definably connected definable abelian group, then $G$ is divisible, i.e., for all $m \in \mathbb{N}$, the definable homomorphism $p_{m}: G \longrightarrow G$ is surjective.

In fact, let $G$ be a definably connected definable abelian group and $m \in \mathbb{N}$. Since $G[m]$ is a finite definable subgroup, $m G$ is a definable subgroup of $G$ of dimension $\operatorname{dim} G$. By [12], $(G: m G)<\aleph_{0}$. But since $G$ is definably connected, again by [12], we must have $m G=G$ as required.

In the paper [7] Margarita Otero observes that with the results from ominimal algebraic topology available in [3] together with classical arguments one can prove that if $\mathcal{N}$ is an o-minimal expansion of a field, then a definably connected, definably compact definable group is divisible. Here we generalise this result in the following way:

Theorem 1.1 If $G$ is a definably compact, definably connected definable group defined in an arbitrary o-minimal structure, then $G$ is divisible. Furthermore, if $G$ is defined in an o-minimal expansion of a field, $k \in \mathbb{N}$ and $p_{k}: G \longrightarrow G$ is the definable map given by $p_{k}(x)=x^{k}$ for all $x \in G$, then for all $x \in G$, we have $\left|\left(p_{k}\right)^{-1}(x)\right| \geq k^{r}$ where $r>0$ is the maximal dimension of abelian definable subgroups of $G$.

The proof of this theorem does not require o-minimal algebraic topology and follows at once from the next Proposition.

Proposition 1.2 Let $G$ be a definably compact, definably connected definable group defined in an arbitrary o-minimal structure. Then there exists a definably compact, definably connected definable abelian subgroup $B$ of $G$ which is unique up to conjugation such that:

(i) $\operatorname{dim} B$ is the maximal dimension of abelian definable subgroups of $G$ and

(ii) $G=\cup\left\{g B g^{-1}: g \in G\right\}$.

From Proposition 1.2 we get Theorem 1.1 in the following way. By Proposition 1.2 , for $x \in G$, there is $b \in B$ and $g \in G$ such that $x=g b g^{-1}$. By 
(DIV) let $a \in B$ be such that $a^{k}=b$. Then $\left(g a g^{-1}\right)^{k}=g a^{k} g^{-1}=g b g^{-1}=x$. Thus $G$ is divisible.

Assume now that $\mathcal{N}$ is an o-minimal expansion of a field and $l=\operatorname{dim} B$. Then by the proof of the structure theorem in [4] (or [3]), the degree of $p_{k}$ in $B$ is $k^{l}$, and so $\left|\left(p_{k}\right)^{-1}(b)\right| \geq k^{l}$ for all $b \in B$. On the other hand, for $x \in G$, by Proposition 1.2, there is $b \in B$ and $g \in G$ such that $x=g b g^{-1}$. By (DIV) let $a \in B$ be such that $a^{k}=b$. Then $\left(g a g^{-1}\right)^{k}=g a^{k} g^{-1}=g b g^{-1}=x$. Thus $\left|\left(p_{k}\right)^{-1}(x)\right| \geq k^{l}$ for all $x \in G$.

For the proof of Proposition 1.2 we require the following claims.

Claim 1.3 Let $G$ be a group, $H$ a subgroup of $G$ and $Z$ a normal subgroup of $G$ contained in $H$ and in the center of $G$. If $G / Z=\cup\left\{g Z(H / Z) g^{-1} Z\right.$ : $g \in G\}$, then $G=\cup\left\{g H g^{-1}: g \in G\right\}$.

Proof. Let $a \in G$. Then there is $g \in G$ and $h \in H$ such that $a Z=$ $g Z h Z g^{-1} Z$. So, there are $z_{1}, z_{2}, z_{3} \in Z$ such that $a=g z_{1} h z_{2} g^{-1} z_{3}$. Since $Z \subseteq Z(G)$, we get $a=g\left(z_{1} h z_{2} z_{3}\right) g^{-1}$. Hence, $G=\cup\left\{g H g^{-1}: g \in G\right\}$ as required.

Claim 1.4 Let $G$ be a group and let $H$ and $K$ be subgroups of $G$ containing a normal subgroup $Z$ of $G$ which is contained in the center of $G$. If $H / Z$ and $K / Z$ are conjugate subgroups of $G / Z$, then $H$ and $K$ are conjugates subgroups of $G$.

Proof. Suppose that $H / Z=g Z(K / Z) g^{-1} Z$. If $h \in H$, then there are $z_{1}, z_{2}, z_{3} \in Z$ and $k \in K$ such that $h=g z_{1} k z_{2} g^{-1} z_{3}$. Since $Z \subseteq Z(G)$ and $Z \subseteq K$, we have $h=g\left(z_{1} k z_{2} z_{3}\right) g^{-1} \in g K g^{-1}$ and $H \subseteq g K g^{-1}$. Similarly, if $k \in K$, then there are $z \in Z$ and $h \in H$ such that $g k g^{-1}=h z \in H$ (since $Z \subseteq H)$ and so $g K g^{-1} \subseteq H$. In conclusion, $H=g K g^{-1}$ as required.

Proof of Proposition 1.2. By [2] Corollary 4.8 or [9] Corollary 5.4, G is abelian or $G / Z(G)^{0}$ is definably semi-simple. In the first case the result follows from (DIV) and the main theorem of [4] (or [3]). So assume that $G / Z(G)^{0}$ is definably semi-simple. The quotient of $G / Z(G)^{0}$ by its finite center is by [10] the direct product $H_{1} \times \cdots \times H_{k}$ of subgroups such that each 
$H_{i}$ is definably isomorphic to an $R_{i}$-semialgebraic subgroup of $\operatorname{GL}\left(n_{i}, R_{i}\right)$ where $R_{i}$ is a real closed field definable in $\mathcal{N}$. Moreover, by the proof of [11] Theorem 5.1, each $H_{i}$ is a definably simple, definably compact, definably connected $R_{i}$-definable group defined over the empty set and so $H_{i}(\mathbb{R})$ is a compact, connected, simple Lie group.

If $T_{i}$ is the maximal torus of $H_{i}(\mathbb{R})$, then $T_{i}$ is definable in $\mathbb{R}$ as the definably connected component of $Z\left(C\left(T_{i}\right)\right.$ ) (using the DCC on definable groups and the fact that any definable group in the field of real numbers is a Lie group). Also, since $\operatorname{dim} T_{i}$ is the maximal dimension of abelian compact Lie subgroups of $H_{i}(\mathbb{R})$, by the definability of o-minimal dimension (see [1]), we can assume that $T_{i}$ is defined over the emptyset. As $H_{i}(\mathbb{R})$ is covered by the conjugates of $T_{i}$, and the real closed field $R_{i}$ is elementarily equivalent to $\mathbb{R}, H_{i}$ is covered by the conjugates (by elements of $H_{i}$ ) of $T_{i}\left(R_{i}\right)$. A similar argument and definability of o-minimal dimension shows that $\operatorname{dim} T_{i}$ is the maximal dimension of abelian definable subgroups of $H_{i}$. Furthermore, if $C_{i}$ is a definably connected definable abelian subgroup of $H_{i}$ such that $\operatorname{dim} C_{i}=\operatorname{dim} T_{i}$, then $C_{i}$ is a conjugate of $T_{i}\left(R_{i}\right)$. Indeed, by definability of o-minimal dimension, there is a first-order formula $\phi(u, v)$ over the empty set such that for every $r \in R_{i}$, the formula $\phi(u, r)$ defines a definably connected definable abelian subgroup of $H_{i}$ of dimension $\operatorname{dim} T_{i}$ and $C_{i}$ is defined by $\phi(u, s)$ for some $s \in R_{i}$. (For the fact that "definably connected" is firstorder see [5] Theorem 0.3). Now since the first-order sentence saying that for all $r$ the subgroup of $H_{i}(\mathbb{R})$ defined by $\phi(u, r)$ is a conjugate of $T_{i}$ is true in $\mathbb{R}$, it must also hold in $R_{i}$. Hence, $C_{i}$ is a conjugate of $T_{i}\left(R_{i}\right)$ as required.

To simplify the notation, we will from now on use $T_{i}$ to denote $T_{i}\left(R_{i}\right)$ for each $i=1, \ldots, k$.

Let $A$ be the minimal (by DCC) definable subgroup of $G / Z(G)^{0}$ such that $A / Z\left(G / Z(G)^{0}\right)=T_{1} \times \cdots \times T_{k}$. Then $\operatorname{dim} \mathrm{A}=\operatorname{dim}\left(T_{1} \times \cdots \times T_{k}\right)$ and by Claim 1.3, G/Z(G) $=\cup\left\{g Z(G)^{0} A g^{-1} Z(G)^{0}: g \in G\right\}$. Note also that $A$ is definably connected. In fact, if $A^{0}$ is the definably connected component of $A$, then by [2] Lemma 3.15 its quotient by the finite center of $G / Z(G)^{0}$ is a definably connected subgroup of $T_{1} \times \cdots \times T_{k}$ of maximal dimension. Thus this quotient is $T_{1} \times \cdots \times T_{k}$ and so $A=A^{0}$.

If $B$ is the minimal definable subgroup of $G$ such that $B / Z(G)^{0}=A$, then $\operatorname{dim} B=\operatorname{dim} Z(G)^{0} \cdot \operatorname{dim} A$ and by Claim 1.3, $G=\cup\left\{g B g^{-1}: g \in G\right\}$. Also by the argument above $B$ is definably connected. Since $B$ is solvable definably compact, it is abelian (by [2] Corollary 4.8 or [9] Corollary 5.4). 
It remains to show that $B$ is unique up to conjugation and $\operatorname{dim} B$ is the maximal dimension of abelian definable subgroups of $G$. Let $B_{1}$ be a definably connected definable abelian subgroup of $G$. By [2] Lemma 3.15, $A_{1}=B_{1} / Z(G)^{0}$ and $A_{1} / Z\left(G / Z(G)^{0}\right)$ are definably connected definable abelian subgroups of $G / Z(G)^{0}$ and $H_{1} \times \cdots \times H_{k}$ respectively. Since $\operatorname{dim} T_{i}$ is the maximal dimension of abelian definable subgroups of $H_{i}$, it follows that $\operatorname{dim} A_{1} \leq \operatorname{dim} A$. So $\operatorname{dim} B_{1}=\operatorname{dim}\left(B_{1} \cap Z(G)^{0}\right) \cdot \operatorname{dim} A_{1} \leq \operatorname{dim} B$.

Assume now that $\operatorname{dim} B_{1}=\operatorname{dim} B$. Then necessarily $\operatorname{dim}\left(B_{1} \cap Z(G)^{0}\right)=$ $\operatorname{dim} Z(G)^{0}$ and $\operatorname{dim} A_{1}=\operatorname{dim} A$. The first condition implies that $Z(G)^{0} \subseteq B_{1}$ and so, by Claim 1.4, we need to show that $A_{1}$ is conjugate to $A$ in order to conclude that $B_{1}$ is conjugate to $B$. For this, for each $i=1, \ldots, k$, consider the projection $C_{i}$ of $A_{1} / Z\left(G / Z(G)^{0}\right)$ into $H_{i}$. By [2] Lemma 3.15, each $C_{i}$ is a definably connected definable abelian subgroup of $H_{i}$. Since $A_{1} / Z\left(G / Z(G)^{0}\right)$ is contained in $C_{1} \times \cdots \times C_{k}$ and $\operatorname{dim} A_{1}=\operatorname{dim} A$, we must have $\operatorname{dim} C_{i}=\operatorname{dim} T_{i}$ for each $i$. Thus, since $C_{1} \times \cdots \times C_{k}$ is definably connected, $A_{1} / Z\left(G / Z(G)^{0}\right)=C_{1} \times \cdots \times C_{k}$. Now as each $C_{i}$ is a conjugate of $T_{i}$, it follows that $C_{1} \times \cdots \times C_{k}$ is a conjugate of $T_{1} \times \cdots \times T_{k}$ and so by Claim $1.4 A_{1}$ is a conjugate of $A$ as required.

\section{References}

[1] L. van den Dries, Tame topology and o-minimal structures Cambridge University Press 1998.

[2] M.Edmundo Solvable groups definable in o-minimal structures J. Pure Appl. Algebra 185 (2003) 103-145.

[3] M.Edmundo O-minimal cohomology and definably compact definable groups RAAG preprint n. 24 (2004) (http://ihpraag.org/).

[4] M.Edmundo and M.Otero Definably compact abelian groups J. Math. Logic 4 (2) (2004) 163-180.

[5] J.Knight, A.Pillay and C. Steinhorn Definable sets in ordered structures II Trans. Amer. Math. Soc. 295 (1986) 593-605. 
[6] J. Krajicek and T. Scanlon Combinatorics with definable sets: Euler characteristics and Grothendieck rings Bull. Symbolic Logic 6 (2000) 311-330.

[7] M.Otero On divisibility in definable groups RAAG preprint $\mathrm{n}$. 147 (2004) (http://ihp-raag.org/).

[8] Y.Peterzil and C.Steinhorn Definable compacteness and definable subgroups of o-minimal groups J. London Math. Soc. 59 (2) (1999) 769-786.

[9] Y.Peterzil and S.Starchenko Definable homomorphisms of abelian groups definable in o-minimal structures Ann. Pure Appl. Logic 101 (1) (1999) 1-27.

[10] Y.Peterzil, A.Pillay and S.Starchenko Definably simple groups in o-minimal structures Trans. Amer. Math. Soc. 352 (10)(2000) 4397-4419.

[11] Y.Peterzil, A.Pillay and S.Starchenko Linear groups definable in o-minimal structures J. Algebra 247 (2002) 1-23.

[12] A.Pillay On groups and fields definable in o-minimal structures J. Pure Appl. Algebra 53 (1988) 239-255.

[13] A.Strzebonski Euler characteristic in semialgebraic and other o-minimal groups J. Pure Appl. Algebra 96 (1994) 173-201. 